\title{
16
}

\section{Benchmarking at Bang \& Olufsen A/S}

\section{J. Bräuner}

\section{Bang \& Olufsen A/S}

Bang \& Olufsen is a medium-size/large company in Denmark. Bang \& Olufsen primary business area is development, production and sales of audio/video products. We sell our products world-wide, however besides our home market the rest of Europe - especially Germany, France and England - is our most important market area.

The company has been through big changes during recent years demanding big efficiencies rises all over.

\section{Why Benchmarking}

At the end of 1992 Bang \& Olufsen decided on a productivity project, and at the same time it was decided that Bang \& Olufsen should use benchmarking as a method of obtaining improvements jumps all over Bang \& Olufsen.

Benchmarking as method was chosen as the method fits very well into the changes that the company is undertaking at the moment. The productivity project causes changes, which result in a much lower organization structure. This means that the individual manager obtains a larger 'span of control', and some of the manager levels are removed, which causes that the lower organization levels are raised. Besides this selfcontrolling production groups are introduced. These groups consist of employees who themselves plan their working day and share out the work. In this respect benchmarking is in a strong position as it is the process owners who take part in the benchmarking project, and it is the process owners who themselves must go out and experience how companies, which carry out performances in the 'Best Practice' class, operate and how they have obtained these performances.

\section{How is benchmarking introduced at Bang \& Olufsen}

In the beginning of the autumn 1993 it was decided to establish a job (full-time) as benchmarking project manager at Bang \& Olufsen. The purpose was to develop the Bang \& Olufsen concept for benchmarking and to describe and introduce benchmarking as method all over at Bang \& Olufsen. This includes preparing of descriptions and instructions which make it possible for other employees in the company to use benchmarking as a method and of course as a method adapted to the Bang \& Olufsen culture and requirements. 
In order of be able to develop the method into a Bang \& Olufsen method it was decided to use a pilot project concerning benchmarking in order to obtain the necessary benchmarking experience.

\section{Choise of benchmarking model:}

As mentioned in various benchmarking literature a benchmarking process course may consist of several steps. We have chosen to divide our first project into 7 phases or steps as follows:

1 Planning. (What should be benchmarked, how, choise of model, coarse planning, team-building)

2 Internal analysis of own process

3 Searching and choise of benchmarking partners

$4 \quad$ Selecting of data from the partners

5 Analysis and adaption of selected results

6 Implementation

7 Follow-up on introduced changes.

\section{Choice of a pilot project}

The choice of a pilot project was dependent on more factors. The pilot project should be very limited, the goals of the project should be realistic and necessary for Bang \& Olufsen as it would facilitate the carrying through of the project, and automatically it would create more motivation in the concerned area. Besides this the area in question should - if possible - be open to changes. This would ease the starting up of the project.

On basis of the above the Bang \& Olufsen Automatical Component Assembling Department (ACAD) was chosen. The goal of the project in the area is a higher exploitation of the assembly of machinery and by this a higher capacity. This means that the focus area of the pilot project is the exploitation degree and as a result of this focus on the loss factors in the area (readjustment time, repair, maintenance, wait between part processes, etc.). One of the main requirements on the ACAD area besides the current requirements concerning quality and costs is the requirement on high flexibility causing smal-scale series.

The ACAD employes approx. 50 - 80 persons dependent on the season and consists of 5-6 partly separated process areas. 


\subsection{Choise of pilot project group}

The signer is project manager of the project group with a co-operation project manager who organizing belongs to the ACAD area. The purpose of a co-operation project manager is partly to give the signer a sparing partner - a person who can review methods, and who in practice selects the members of the project group.

It is very important that the members of the project group are process owners or representative for these - this is of essential importance to the success of the benchmarking project.

In connection with the selection of the participants in the project group the first problems arose. The persons that we wanted to take part in the project were the persons who already had a huge working pressure on them, and therefore they were not able to spare time for participation in a benchmarking project in which the biggest problem was release of machine operators. After some internal discussions in the area internal resources were removed from another area to the ACAD area. This meant that sufficient resources could be released for the benchmarking project. It also meant that the benchmarking project received very good support from the top management. It is a very good motivation for the project members that they can see that the top management also finds the project important.

In order to get the processes of the whole area represented the project group has become rather big. The reason of this is that we want to have all the process owners directly represented in the project group.

In connection with the starting up of the project the project group has taken part in the selection of a working structure. The project group has been divided into two - a SMD group and a group for the leaded assembly, which then at suitable intervals coordinate their points of views. The division is very clearly in the first phases, and at the end in the implementation phase. The division has been chosen in order to obtain a higher process time. Crosswise of the two groups a smaller group exists, which takes part in the work of both 'main groups'. This also applies to the project management. The structure is shown in fig. 1 .

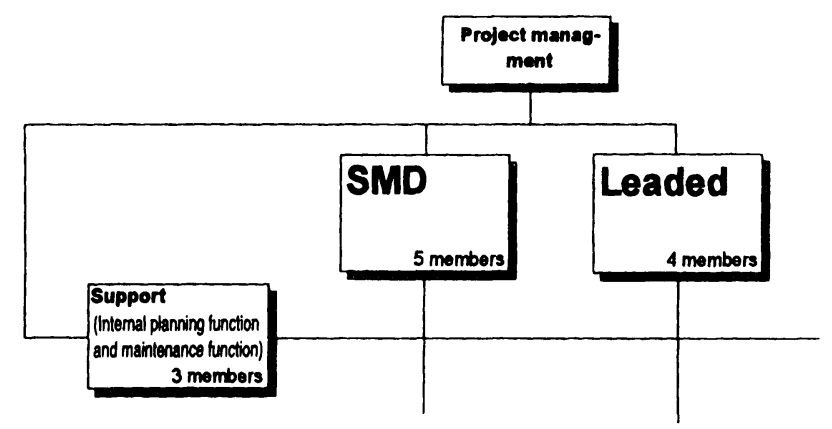

Fig. 1 


\section{The Benchmarking Course}

Below the rough plan of the project including the planned terms and real terms is shown.

\begin{tabular}{lcc}
\multicolumn{1}{c}{ Phase: } & $\begin{array}{c}\text { Planned } \\
\text { (end time) }\end{array}$ & $\begin{array}{c}\text { Real } \\
\text { (end time) }\end{array}$ \\
\hline 1: Planning phase with team-building & $02 / 94$ & $03 / 94$ \\
2: Internal analysis of own process & $08 / 94$ & $11 / 94$ \\
3: Searching and choice of partners & $14 / 94$ & $22 / 94$ \\
4: Selecting of data from the partners & $22 / 94$ & $34 / 94$ \\
5: Analysis and adaptation of selected resultss & $27 / 94$ & $37 / 94$ \\
6: Implementation & $35 / 94$ & $41 / 94$ \\
7: Follow-up & approx. 1/2 & approx. 1/2 \\
& year & year \\
\hline
\end{tabular}

At present in the middle of August 1994 we are in the end of phase 4, "Collecting of data". Compared to the original rough plan we are therefore delayed some weeks due to various courses.

\section{Experiences achieved via the pilot project}

In the following I shall touch on some of the experiences that we/I have had until now in connection with our pilot project:

\subsection{The project group}

The composition of the participants of the project group was made from the point of view that all process owners should be represented if possible. However, this meant that we got a fairly big group. Due to the size of the group we thought it expedient to divide the work into two groups, who each of them dealt with two partly separated main process areas. As it is shown in fig. 1 some of the participants participated in both sub-project groups - e.g. the signer of course. The experiences from this sub-division and size is today of a doubtful character, but as in the main the following can be mentioned:

- Diffecult to coordinate efforts between the sub-groups

- Big strain on some of the participants - the participants who were in both groups started (more or less) in one of the sub-groups then in the other, which caused lack of teamwork - the group as a total became big and very difficult to work with.

- Many sub-process owners were participants in the project group.

Today I would choose a smaller firm group of max. 6-7 people and after than maybe include other ad hoc in the analysis phase. 
On basis of this we decided to reduce the group from the 12 people to 8 people in total having in mind that those remaining should work a little harder. This group has now be working since the beginning of May, and I find it a better and more dynamic group.

\subsection{Time schedule}

As earlier mentioned the time schedule was only a rough outline, and I had no experience about how long time things take, and maybe therefore we are also delayed compaired to the original plan. A delay, which I do not find dramatical, remember it is the first time we are running a benchmarking project - and that we find that quality in benchmarking should have a higher priority than keeping the time schedule. However, there is one thing you should be aware of as project manager of benchmarking project - things which apply to all kinds of project work in which the participants of the project group are not full-time members - and it is very unusual that the members are full-time members especially at Bang \& Olufsen. Often it will be the same persons, who take part in various projects/tasks at the same time - as it always will be the best employees who are chosen for these kind of tasks, and thereby it will often be the same employees.

\subsection{Contact to external companies:}

Because this was our first benchmarking project I decided that we not necessarily would look for "Best Practice" companies, but only for companies from which we could learn and from companies which were rather easily accessible - that is Danish companies. The final choice became two Danish and one Norwegian company.

Our way of choosing the companies was made by means of a gross list including potential partners. These were chosen from our internal network, suppliers, organizations and literature references. Among the listed potential companies (>50) approx. 15 were chosen. At these companies we would seak extra information, primarily by means of a questionnaire sent out to them.

To make a questionnare which gives you information about "How good is company $X$ at...." is very difficult, especially when it is the first time. It took much longer than anticipated. We used at least 5 working weeks for making our questionnaire, a questionnare which is not at all the best questionnaire, however, it is a start, and I think it is a questionnaire which may form the basis of making questionnaires for the benchmarking projects at Bang \& Olufsen. Together with the questionnaire I included a letter which told about benchmarking, the purpose of the application, and with information about us.

\subsection{Benefits until now}

What have we achieved until now:

- Establishing of a mutual measuring system and establishing of a number of measurings of the coefficient of utilization, on which we can make a follow-up.

- A number of "Entitlements", which we - compaired with results of the data collection at our partners - should have implemented.

- That the various levels/areas in the project work speak together much more than before (activation of employees).

- An almost finished method which, however, should be reviewed one more time. 


\subsection{What next}

As mentioned earlier I am responsible for disseminating the method benchmarking all over at Bang \& Olufsen. This is done by starting up projects in the individual areas of the company. When a project is started up a "cooperation project manager" is chosen as in the pilot project case. This person belongs to the area in which benchmarking should take place. This means that at least one person in the area after this should be able to carry out benchmarking projects. By choosing this method knowledge about and owner-ship of the method is obtained in the individual areas of the company. The owner-ship implies of course that the first project in the area is a success. Furthermore information to the whole organization must take place constantly concerning benchmarking and how the individual projects go and which results are achieved. Parallel with the starting up of the project a "benchmarking knowledge center" is built up consisting of the signer. This knowledge center should take care of:

- Building up of a data base with data about companies, which have processes of "Best practice" level.

- Collecting of data to the data base.

- Maintenance of the method.

- Follow-up and registration of results achieved at Bang \& Olufsen.

- Take care of forwarding benchmarking newsletters in the company.

- Facilitator of new projects and project groups.

- Consultant for other project groups. 